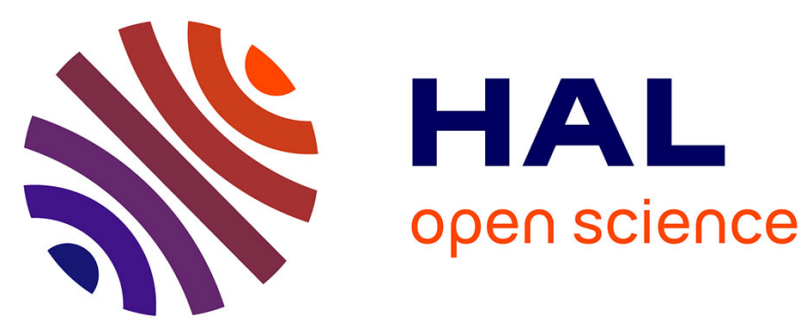

\title{
Dark current investigation in thin P-i-N InGaAs photodiodes for nano-resonators
}

Michael Verdun, Grégoire Beaudoin, Benjamin Portier, Nathalie Bardou, Christophe Dupuis, Isabelle Sagnes, Riad Haïdar, Fabrice Pardo, Jean-Luc Pelouard

\section{To cite this version:}

Michael Verdun, Grégoire Beaudoin, Benjamin Portier, Nathalie Bardou, Christophe Dupuis, et al.. Dark current investigation in thin P-i-N InGaAs photodiodes for nano-resonators. Journal of Applied Physics, 2016, 120 (8), pp.084501. 10.1063/1.4961327 . hal-01717473

\section{HAL Id: hal-01717473 https://hal.science/hal-01717473}

Submitted on 26 Feb 2018

HAL is a multi-disciplinary open access archive for the deposit and dissemination of scientific research documents, whether they are published or not. The documents may come from teaching and research institutions in France or abroad, or from public or private research centers.
L'archive ouverte pluridisciplinaire HAL, est destinée au dépôt et à la diffusion de documents scientifiques de niveau recherche, publiés ou non, émanant des établissements d'enseignement et de recherche français ou étrangers, des laboratoires publics ou privés. 


\section{Dark current investigation in thin P-i-N InGaAs photodiodes for nano-resonators}

Michaël Verdun, Grégoire Beaudoin, Benjamin Portier, Nathalie Bardou, Christophe Dupuis, Isabelle Sagnes, Riad Haïdar, Fabrice Pardo, and Jean-Luc Pelouard

Citation: Journal of Applied Physics 120, 084501 (2016);

View online: https://doi.org/10.1063/1.4961327

View Table of Contents: http://aip.scitation.org/toc/jap/120/8

Published by the American Institute of Physics

\section{Articles you may be interested in}

Guided-mode resonator for thin InGaAs P-i-N short-wave infrared photo-diode Applied Physics Letters 108, 053501 (2016); 10.1063/1.4941297

2.6 $\mu \mathrm{m}$ MBE grown InGaAs detectors with dark current of SRH and TAT AlP Advances 4, 087135 (2014); 10.1063/1.4894142

Impact of $\mathrm{SiN}_{\mathrm{X}}$ passivation on the surface properties of InGaAs photo-detectors Journal of Applied Physics 118, 034507 (2015); 10.1063/1.4926736

Comparison of intrinsic width for InGaAs PIN photodiode AIP Conference Proceedings 1586, 147 (2014); 10.1063/1.4866750

Optical index measurement of InAs/GaSb type-II superlattice for mid-infrared photodetection at cryogenic temperatures

Applied Physics Letters 105, 141103 (2014); 10.1063/1.4897273

Effect of surface charge on the dark current of InGaAs/InP avalanche photodiodes Journal of Applied Physics 115, 164512 (2014); 10.1063/1.4874178

\section{Scilight} Sharp, quick summaries illuminating the latest physics research

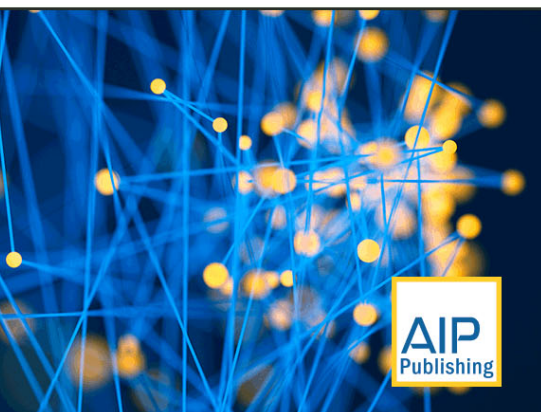




\title{
Dark current investigation in thin P-i-N InGaAs photodiodes for nano-resonators
}

\author{
Michaël Verdun, ${ }^{1,2}$ Grégoire Beaudoin, ${ }^{3}$ Benjamin Portier, ${ }^{1}$ Nathalie Bardou, ${ }^{1}$ \\ Christophe Dupuis, ${ }^{1}$ Isabelle Sagnes, ${ }^{3}$ Riad Haïdar, ${ }^{2,4}$ Fabrice Pardo, ${ }^{1}$ \\ and Jean-Luc Pelouard ${ }^{1, a)}$ \\ ${ }^{1} \mathrm{MiNaO}$ - Center for Nanoscience and Nanotechnology (C2N) - CNRS, University of Paris-Sud, \\ Université Paris-Saclay, 91460 Marcoussis, France \\ ${ }^{2} \mathrm{MiNaO}$ - ONERA, the French Aerospace Lab, Chemin de la Hunière, Université Paris-Saclay, \\ F-91760 Palaiseau, France \\ ${ }^{3}$ Center for Nanoscience and Nanotechnology (C2N) - CNRS, University Paris-Sud, Université Paris-Saclay, \\ 91460 Marcoussis, France \\ ${ }^{4}$ Département de Physique, École Polytechnique, Université Paris-Saclay, F-91128 Palaiseau, France
}

(Received 23 June 2016; accepted 7 August 2016; published online 22 August 2016)

\begin{abstract}
We investigated the dark current components of thin planar InGaAs photodiodes grown by metalorganic vapor-phase epitaxy for optical nano-resonators. Owing to their high electric field enhancement, nano-resonators make it possible to substantially reduce the thickness of the active region to about $100 \mathrm{~nm}$ all the while maintaining high quantum efficiency. In the present study, to cover a broad spectral band, we combined several resonance peaks induced by guided-mode resonances in a given spectral range. This type of geometry allowed us to introduce InAlAs at the edge of a thin InGaAs active region in order to drastically reduce both the diffusion current and the generation/recombination current. We found that, in such devices, tunneling dark current components increase as the thickness of the active layer is reduced and dominate the reverse dark current. By optimizing the epitaxial stack, while keeping its total thickness constant (the optical properties of the nano-resonator remained unchanged), we showed that we are already able to achieve a specific detectivity of up to $1 \times 10^{13} \mathrm{~cm} \sqrt{\mathrm{Hz}} \mathrm{W}^{-1}$ for $\lambda=1.55 \mu \mathrm{m}$. Published by AIP Publishing.

[http://dx.doi.org/10.1063/1.4961327]
\end{abstract}

\section{INTRODUCTION}

Since the invention of the first infrared detectors in the mid-1960s, there have been three generations of detectors. ${ }^{1,2}$ To improve the performances of today's infrared focal plane array (FPA) geometries and add new functionalities, researchers are currently working on a fourth generation of detectors. ${ }^{3}$ Although they have yet to be clearly defined, we already know that they will feature a promising semiconductor stack that includes barrier structures and nanophotonics concepts such as plasmonic resonances. They are expected to exhibit enhanced capabilities such as higher operating temperatures, smaller pixels, higher frame rates, multicolor functionalities, and others on chip functions.

Of all the detectors that can be used in the short-wave infrared (SWIR) domain, InGaAs photodiodes have the best performances because of their high efficiency and low dark current at room temperature. ${ }^{4}$ In this spectral range, they can be used for imaging through haze or under Nightglow illumination. ${ }^{5}$ Because the light flux is low in these conditions, detectors need to have the highest possible detectivity, while that of InGaAs detectors is mainly limited by the noise generated by the dark current. ${ }^{6}$ Despite the outstanding performances achieved by today's InGaAs detectors, there have been few advances in recent years. ${ }^{7-9}$

SWIR InGaAs photodiodes can be seen as a model for the development of the fourth generation of infrared detectors.

a) jean-luc.pelouard@1pn.cnrs.fr
Combining an optical resonator with a semiconductor structure that features both a double heterojunction and a thin absorbing layer, they allow the dark current to be drastically reduced, all the while maintaining high quantum efficiency. ${ }^{10}$ This design has been put into practice since the early 1990s for SWIR light-wave communication by inserting an InGaAs photodiode in a Fabry-Perot cavity formed by two parallel mirrors. ${ }^{11-14}$ However, the bandwidth of these resonators is too small for SWIR imaging applications. This is because quantum efficiency tends to decrease rapidly outside the resonance bandwidth, as large field enhancement is required in the cavity to achieve external quantum efficiency (EQE) close to unity despite the thin absorbing layer. Moreover, these detectors have mainly been developed for their high-speed behavior rather than for their low dark current, despite Sverdlov's pioneering paper $^{10}$ providing proof of concept for dark-current reduction in a resonant cavity.

In this article, we investigate the components of the dark current in thin $\mathrm{P}-\mathrm{i}-\mathrm{N}$ InGaAs photodiodes within an optical resonator and showed that the tunneling components exhibit optimum detectivity for an absorbing layer thickness of a few hundred nanometers. We begin by describing the optical resonator, a nanostructured guided-mode resonator (GMR) that combines several resonance peaks within a given spectral range. This device is made up of a single crystal active epilayer transferred onto a gold mirror, and a subwavelength dielectric grating is inserted between the mirror and the detector. We then explain how we investigated the dark 
current components of this detector, grown by metalorganic vapor-phase epitaxy (MOVPE). The semiconductor structure comprises an InGaAs/InAlAs double heterojunction with a reduced active region thickness of about $100 \mathrm{~nm}$. Our best structure achieved a volume dark current density of just $20 \mathrm{nA} \mathrm{cm}^{-2}$ at $-100 \mathrm{mV}$, resulting in a specific detectivity of up to $1 \times 10^{13} \mathrm{~cm} \sqrt{\mathrm{Hz}} \mathrm{W}^{-1}$ for $\lambda=1.55 \mu \mathrm{m}$.

\section{DETECTOR DESIGN}

\section{A. Guided mode resonator}

Traditional InGaAs Fabry-Perot detectors, which have been around since the mid-1980s, are formed by a semiconductor active layer placed between two parallel mirrors. ${ }^{1-14}$ In these resonators, the bandwidth is reduced as the electric field is enhanced. This is well suited to light-wave communication, but imaging usually requires a much larger bandwidth, especially for low-incident flux. In order to achieve a wider spectral response, we chose to create several resonances in a given spectral range, using a GMR. This structure consists of a semiconductor stack and a backside subwavelength grating on a gold mirror (see Fig. 1). Its design allows all the nanofabrication steps to be achieved prior to the layer transfer, making the fabrication process compatible with the indium bonding technique for FPA production. Moreover, the dielectric grating is entirely located in the highly p-doped layer to avoid any electrical activation of additional traps resulting from grating fabrication.

Using a B-Spline modal method, ${ }^{15}$ the structural parameters (see Fig. 1) were optimized to maximize a main resonance around $\lambda=1.55 \mu \mathrm{m}$ for both polarizations. As InP, InAlAs, and InGaAs have similar refractive indices, the whole semiconductor heterostructure acts as a single waveguide that is limited by the semiconductor air interface and the semiconductor gold interface. We showed in a previous study $^{16}$ that propagation inside the semiconductor cavity involves modes coming from combinations of diffracted waves of several orders. We also observed that this configuration makes it possible to achieve near perfect collection of the photo-carriers. Figure 2 shows the measured EQE of a typical InGaAs GMR detector for an unpolarized focused beam as a function of wavelength. Over the $1.2-1.8 \mu \mathrm{m}$,

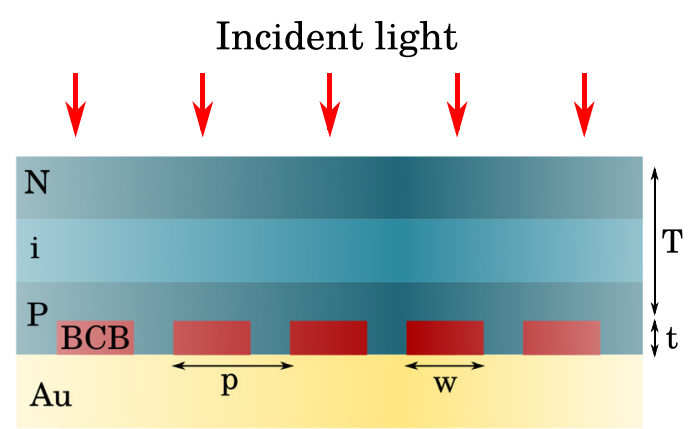

FIG. 1. Schematic cross-section showing five periods of the device. P-i-N photodiode of total thickness $T=387 \mathrm{~nm}$ on top of a backside semiconductor/dielectric grating of period $p=1100 \mathrm{~nm}$, width $w=220 \mathrm{~nm}$, and thickness $t=200 \mathrm{~nm}$ on a gold mirror. The benzocyclobutene (BCB) dielectric used for the grating is a resist that is commonly used in microfabrication.

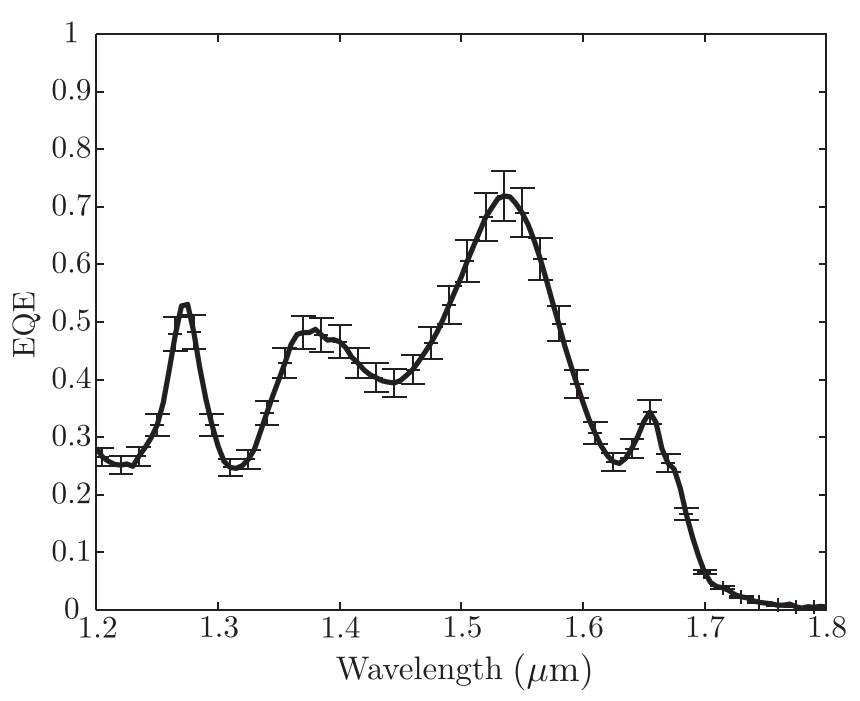

FIG. 2. Measured external quantum efficiency of an InGaAs GMR detector for an unpolarized focused beam as a function of wavelength.

the EQE had a maximum value of $70 \%$ at $\lambda \simeq 1.55 \mu \mathrm{m}$ and a mean value of about $50 \%$ over a $450-\mathrm{nm}$ bandwidth.

\section{B. Semiconductor stack}

$\mathrm{P}-\mathrm{i}-\mathrm{N}$ junctions are the most commonly used semiconductor structures in infrared detection. Owing to their high impedance under reverse bias, they are well suited to the mode amplifiers that are currently utilized in silicon read-out integrated circuits for an imaging application.

In a P-i-N homojunction, the main contribution to the dark current comes from the diffusion current. It is defined by the gradient of minority carriers in the two quasi-neutral regions (QNRs). In these thin layers, gradients are mainly defined by the boundary conditions at each end. Under reverse bias conditions, minority carrier density tends toward zero at the edge of the space charge region (SCR). Thus, the current saturates to a value defined solely by QNR thickness and the second boundary condition. The latter can then be drastically reduced by varying the surface recombination velocity or introducing a wider band-gap semiconductor.

In the field of imaging InGaAs detectors, a low dark current heterostructure made up of a P-n InP/InGaAs junction on an InP substrate was developed in the 1980s. ${ }^{17-20}$ Although sometimes referred to as a P-i-N double heterojunction structure, this stack actually behaves as a single heterojunction, located on the p-side. Owing to the excessive thickness of the absorption layer, the n-border of the SCR is located in the InGaAs layer. To achieve high quantum efficiency over the widest possible spectral range, the absorption region needs to be several micrometers thick. At low reverse bias, complete depletion of the InGaAs layer is therefore not possible. The diffusion current of minority carriers in the QNR is therefore defined by the transport conditions imposed on the InGaAs material. The dark current levels attained by detectors fabricated today are mainly limited by minority carrier diffusion in the n-region of the junction. ${ }^{8}$ Improving the performance of these detectors is a long and difficult task that involves reducing this current component 
by modifying the surface recombination velocities. This is achieved by optimizing the n-doping profile and geometry of the FPAs. ${ }^{8,21}$ With the best devices, dark current densities can be as low as a few $\mathrm{nA} \mathrm{cm}{ }^{-2}$ at ambient temperature. ${ }^{7-9}$

Optical nano-resonators allow us to substantially reduce the absorbing layer thickness while maintaining high absorption. In this context, we can introduce a heterojunction on either side of the SCR, thus dramatically reducing the diffusion current components. At ambient temperature, InAlAs has a bandgap energy of $1.45 \mathrm{eV}$, that is $710 \mathrm{meV}$ larger that of InGaAs. ${ }^{22}$ This difference allows the diffusion components to be reduced by 12 orders of magnitude. The diffusion dark current is proportional to $n_{i}^{2}$ (i.e., $e^{\frac{E_{g}}{k_{b} T}}$, where $E_{g}$ is the semiconductor's bandgap cutoff). The diffusion dark current reduction can then be approximated by $e^{\frac{\Delta E_{g}}{k_{b} T}} \simeq 10^{12}$. In an InAlAs/InGaAs/InAlAs double heterostructure, the only remaining current component arises from generation/recombination $(\mathrm{G} / \mathrm{R})$ mechanisms. G/R currents can be reduced by cutting the SCR thickness by one order of magnitude. With this geometry, we would expect to reduce the dark current by at least one order of magnitude compared with the state of the art of InGaAs photodiodes. ${ }^{7-9}$

Being the simplest material in this system, InP is a good candidate as the wide bandgap material in these heterojunctions. Unfortunately, it has two major drawbacks for InGaAs/InP stacks grown by MOVPE. First, the interfaces are not well defined because of the substitution of arsenic by phosphorus atoms in subsurface InGaAs monolayers. ${ }^{23}$ That said, InP/InGaAs interfaces are much more abrupt than inverted ones. ${ }^{23}$ Second, in InP, the element (zinc, $\mathrm{Zn}$ ) used for p-type doping during growth is known to diffuse with a diffusion length of up to several hundred nanometers. ${ }^{24}$ Because flaws introduced by these two mechanisms would strength the Shockley-Read-Hall (SRH) currents, we introduced an InAlAs layer at each SCR edge. InGaAs/InAlAs interfaces are very well defined, ${ }^{23}$ and $\mathrm{Zn}$ has an abrupt profile in InAlAs. ${ }^{25}$

The epitaxial stack of a typical junction dedicated to the GMR is illustrated in Table I. The InGaAs layer in contact with the substrate is an etch-stop layer for wet substrate removal. The next layer is also an etch-stop layer for

TABLE I. Epitaxial stack A.

\begin{tabular}{|c|c|c|c|}
\hline Layer & Material & Doping $\left(\mathrm{cm}^{-3}\right)$ & Thickness (nm) \\
\hline $\mathrm{P}$ contact & InGaAs & $\mathrm{Zn}: 5 \times 10^{18}$ & 20 \\
\hline $\mathrm{P}$ barrier & $\operatorname{InP}$ & $\mathrm{Zn}: 2 \times 10^{18}$ & 170 \\
\hline Etch stop layer & InGaAs & $\mathrm{Zn}: 2.5 \times 10^{18}$ & 10 \\
\hline $\mathrm{P}$ barrier & $\mathrm{InP}$ & $\mathrm{Zn}: 2 \times 10^{18}$ & 40 \\
\hline $\mathrm{P}$ barrier & InAlAs & $\mathrm{Zn}: 2 \times 10^{18}$ & 43 \\
\hline Spacer & InAlAs & Nid & 10 \\
\hline Active region & InGaAs & $\mathrm{Nid}$ & 90 \\
\hline $\mathrm{N}$ barrier & InAlAs & Si: $3 \times 10^{18}$ & 164 \\
\hline $\mathrm{N}$ barrier & $\mathrm{InP}$ & Si: $5 \times 10^{18}$ & 40 \\
\hline $\mathrm{N}$ contact & InGaAs & Si: $3 \times 10^{18}$ & 10 \\
\hline Etch stop layer & InP & $\mathrm{Si}:>5 \times 10^{18}$ & 100 \\
\hline Etch stop layer & InGaAs & Si: $>3 \times 10^{18}$ & 100 \\
\hline Substrate & InP & $\ldots$ & $\ldots$ \\
\hline
\end{tabular}

removing the InGaAs etch-stop layer. The remaining layers are the device's active layers. The InGaAs layers on either side of the junction serve as contact layers. The layers (barriers), which are useful for reducing diffusion currents, are located on either side of the active layer in InGaAs. The top InGaAs etch-stop layer is used to precisely control the depth of the dielectric grating (see Ref. 16 for more information about the GMR nanofabrication process). As a result, the 200-nm deep grating is entirely located in the p-doped region. An undoped 10-nm thick InAlAs spacer is introduced at the p-type InGaAs/InAlAs interface to ensure an abrupt doping profile there.

\section{EXPERIMENTAL REALIZATION}

\section{A. Sample fabrication}

The state-of-the-art InGaAs detector used for infrared imaging comprises a PN junction fabricated by local $\mathrm{Zn}$ diffusion. ${ }^{17-20}$ This diffusion is difficult to manage for thin junctions, where the diffusion depth must be precisioncontrolled to within a few tens of nanometers. It is therefore preferable for the thin junctions to be entirely formed during the epitaxial growth. In this study, the epitaxial layers were grown by MOVPE in a VEECO TurboDisc D180 system on n-doped InP substrates at $630^{\circ} \mathrm{C}$.

To characterize the dark currents associated with these semiconductor stacks, we fabricated devices of different dimensions. They were specifically designed diodes for measuring dark current: the upper electrode covered the whole device area, meaning that no external light flux could be absorbed. Fabrication took place in three steps. First, a layer of $\mathrm{Ti} / \mathrm{Au}(20 / 200 \mathrm{~nm})$ was deposited on the backside of the substrate in order to make the common n-contact. Then, square-shaped contacts made of $\mathrm{Ti} / \mathrm{Au}(20 / 200 \mathrm{~nm})$ were patterned on the p-doped side using a lift-off technique with optical lithography. Finally, resin masks were patterned on these contacts by optical lithography, to chemically etch mesas down to the n-region. The InP layers were etched in a hydrochloric acid solution, whereas the InAlAs and InGaAs materials was etched in an orthophosphoric acid/hydrogen peroxide solution.

At the end of the fabrication process, one hundred square-shaped diodes of five geometries (side of 50, 65, 100, 150 , and $300 \mu \mathrm{m}$ ) were available on each of the six samples (see Fig. 3). These different geometries would allow us to extract the volume dark current density of the epitaxial stacks.

\section{B. Dark-current characterization}

The dark current was measured with a sourcemeasurement unit (Keithley 236). For a -1 to $+1 \mathrm{~V}$ voltage range, the dark current typically varies from $10^{-12}$ to $1 \mathrm{~A}$.

The volume current characterizes the epitaxial stack, whereas the peripheral current is related to the device fabrication (i.e., mainly due to mesa etching). Writing $I(U)$ for the dark current measured at a given voltage $U, A$ for the device area, and $P$ for its periphery, they can be expressed as 

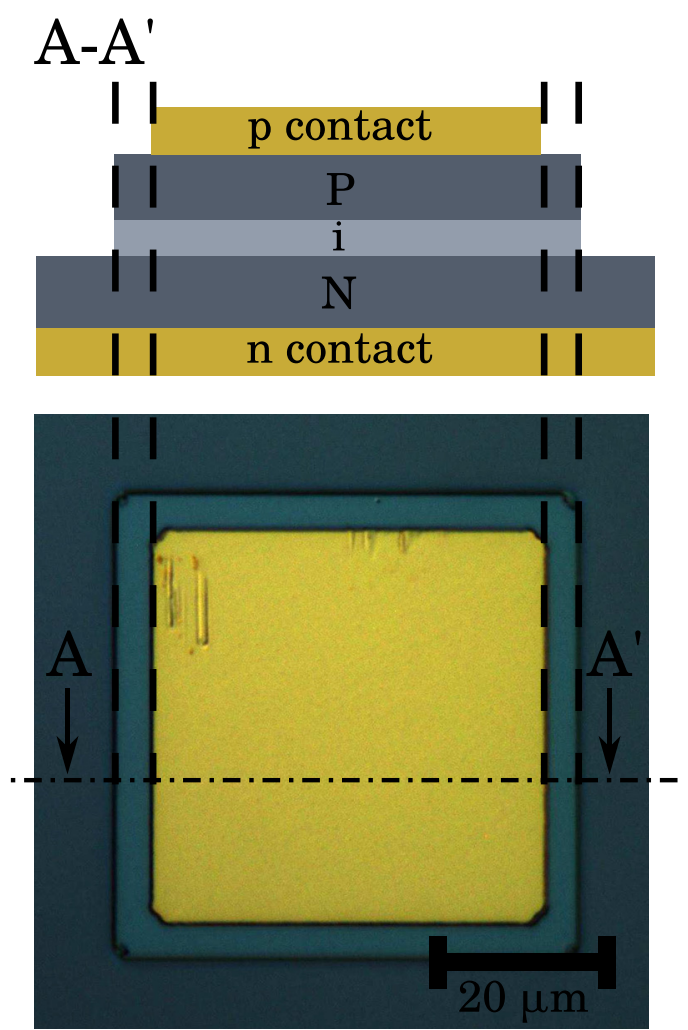

FIG. 3. Top: Schematic cross-sectional view of a $50 \times 50 \mu$ m diode along $\left(\mathrm{AA}^{\prime}\right)$. Bottom: Optical microscope image of this device (top view).

$$
\frac{I(U)}{A}=j_{v}(U)+j_{p}(U) \times \frac{P}{A},
$$

where $j_{v}$ is the volume dark current density and $j_{p} \times \frac{P}{A}$ the peripheral dark current density. The two current densities $\left(j_{v}\right.$ and $j_{p}$ ) were extracted for each bias voltage from device populations of about 100 devices per geometry. At a given bias voltage, mean value $(\langle I\rangle)$ and standard deviation were calculated for the measured current in each geometry. The $\frac{\langle I\rangle}{A}$ as a function of $\frac{P}{A}$ was then fitted to Equation (1) using $j_{v}$ and $j_{p}$ as free parameters and standard deviation as the weighted reliability factor (see Fig. 4). By so doing, for each bias voltage value, we were able to extract $j_{v}(U)$ and $j_{p}(U)$ characteristics, yielding mean value and uncertainty for each bias voltage.

Figure 4 shows that the peripheral dark current can be much larger (about one order of magnitude for a square device of about $30 \times 30 \mu \mathrm{m}$ ) than the volume dark current density. Therefore, further technological efforts are needed to reduce this current component for FPA production. Benzocyclobutene (BCB) passivation has brought about tenfold improvement for similar structures in this field. ${ }^{26}$ In our study, we only investigated volume current characteristics in order to evaluate semiconductor stack performances.

\section{DISCUSSION}

\section{A. Initial measurement and modeling}

The black line with error bars in Figure 5 shows the volume dark current density of the epitaxial stack A (see Table I), as extracted with the algorithm described above.

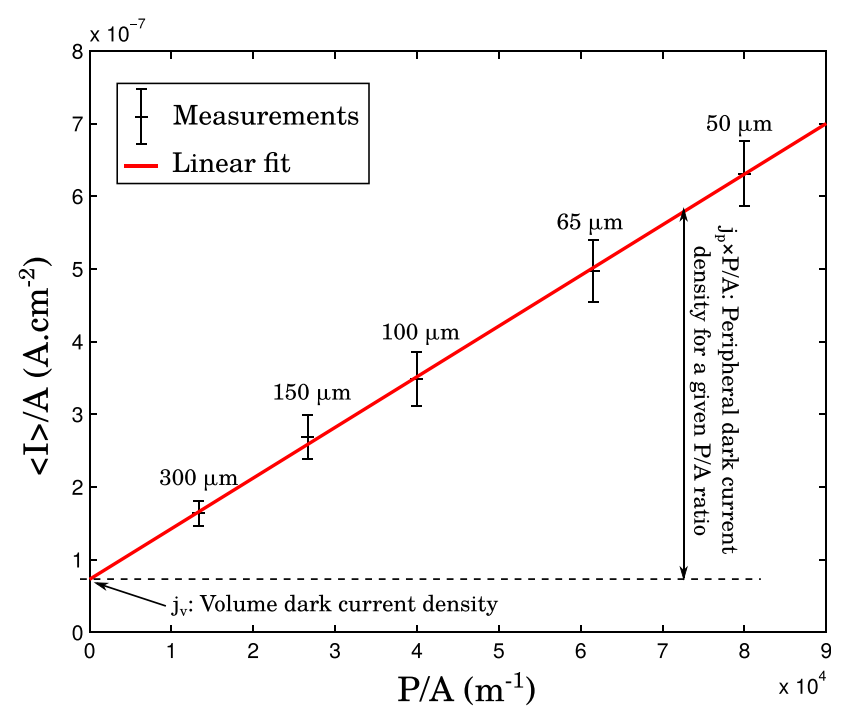

FIG. 4. Example of volume and peripheral dark current density extraction for voltage $U=0.1 \mathrm{~V}$.

We observed conventional behavior for the forward bias voltage, where the current grew exponentially with the voltage. For the highest direct bias voltage $(U>0.2 \mathrm{~V})$, the ideality factor was equal to 1 . This current mainly came from the radiative recombinations in the SCR. Introducing a double heterojunction drastically reduced the diffusion current. Thus, the forward dark current arose from the recombination in the SCR. It could be either non-radiative (SRH recombination) or radiative. Radiative recombination dominates nonradiative recombination for the highest bias voltage. It can be expressed as

$$
j_{\text {rad }}(U)=j_{\text {rad }}^{s}\left(\exp \left(\frac{q U}{k_{b} T}\right)-1\right) .
$$

In the reverse bias voltage, we observed excess current with two different components. This behavior is indicative of a tunneling effect. As in Ref. 27, this tunneling seems to arise from band-to-band tunneling for the highest reverse

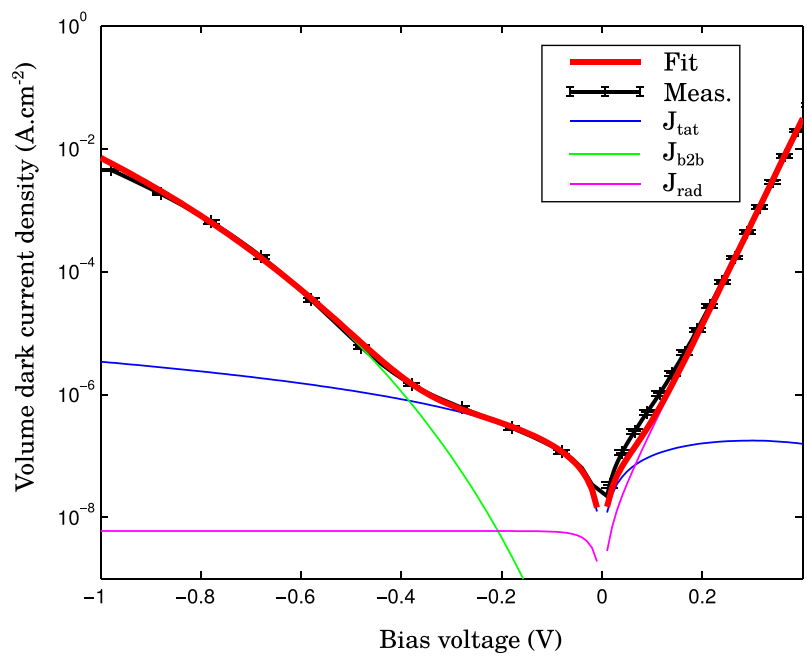

FIG. 5. Volume dark current density of the epitaxial stack A. 
bias voltages $(U<-0.4 \mathrm{~V})$, and trap-assisted tunneling for the lowest ones $(0 \mathrm{~V}>U>-0.4 \mathrm{~V})$.

$\mathrm{Kane}^{28}$ showed that band-to-band tunneling current $j_{b 2 b}$ can be written as

$$
j_{b 2 b}(U)=\alpha F U\left(\exp \left(\frac{-\beta}{F}\right)\right),
$$

where $F$ is the constant electric field in the SCR, and $\alpha$ and $\beta$ are the two parameters to determine in accordance with the experimental data.

The trap-assisted tunneling current $j_{\text {tat }}$ is nothing more than SRH generation enhanced by tunneling effects. In the literature, there are two main approaches to modeling this current. SRH recombination involves only thermal transitions. To evaluate the trap-assisted tunneling, the first approach comprises evaluating all the other transition paths for each trap: thermal-tunnel transitions, tunnel-thermal transitions, and tunnel-tunnel transitions. ${ }^{29}$ The second approach comprises reducing carrier lifetime by taking a tunneling factor into account in the standard SRH generation/recombination rate. ${ }^{30}$ The trap-assisted tunneling current therefore depends on many parameters, including trap distribution in both space and energy. In the present paper, we consider a model where the tunneling mechanism is dominant (i.e., the tunneling mechanism has the longest constant time), which allows us to assume that this current behaves the same as that of band-to-band tunneling (see Eq. (3)). This very simple approach (only two parameters to be determined) accurately models our experimental data.

Finally, volume dark current density can be written as the sum of three contributions

$$
j_{v}=j_{r a d}+j_{t a t}+j_{b 2 b}
$$

where $j_{\text {rad }}$ is the current associated with the radiative generation/recombination in the SCR, $j_{\text {tat }}$ is the trap-assisted tunneling current, and $j_{b 2 b}$ is the band-to-band tunneling current. The parameters of Equation (4) were determined to fit the experimental values of Sample A (see red line in Fig. 5). The parameters are set out in Table II.

If we consider Van Roosbroeck and Schockley's radiative lifetime model, ${ }^{31}$ we can write $j_{\text {rad }}^{s} \simeq q B n_{i}^{2} W$, where $B$ is the bimolecular recombination coefficient of the semiconductor, $n_{i}$ is its intrinsic carrier density, and $W$ is the width of the SCR. If we take parameters $B$ and $n_{i}$ from Ref. 32, we obtain $j_{\text {rad }}^{s}=5 \times 10^{-11} \mathrm{~A} \mathrm{~cm}^{-2}$. This value is two orders of magnitude below that obtained by fitting the experimental data. This can be explained by the photon recycling effect pointed out by Humphreys: ${ }^{33,34}$ reabsorption is drastically reduced in a thin material layer compared with a thicker one; therefore, in a bulk material, lifetime increases linearly with thickness. This explains why we can find a lifetime two orders of magnitude smaller in a $100 \mathrm{~nm}$-thick layer than in bulk material.

At the lowest forward bias voltages $(0 \mathrm{~V}<U<0.2 \mathrm{~V})$, our experimental data indicated slight excess current with respect to the radiative contribution (see magenta line in Fig. 5). This can be explained partly by a tunneling current (see blue line in Fig. 5), and partly by another current component that was not extracted, owing to a lack of precision. This may have been either a leakage current (parallel resistance) or an SRH current.

In reverse bias, our model was in very good agreement with the experimental values. The assumption of the tunneling effect is supported below, where we discuss the effect of SCR thickness.

The dark current of Sample A was thus accurately modeled by the three components of Equation (4). We then turned our attention to the influence of growth temperature and active region thickness on each component, in terms of reducing the dark current.

\section{B. Growth temperature}

Temperature is a key parameter for MOVPE growth. We chose the growth temperature of $630^{\circ} \mathrm{C}$ to reduce $\mathrm{Zn}$ diffusion length. It should be noted that the active region was obtained at the higher temperature of $675^{\circ} \mathrm{C}$ for all samples except Sample A. The volume dark current densities of Samples A and B are plotted in Figure 6.

A notable change was only observed for the lowest forward bias voltages: the excess current observed on Sample A was not present on Sample B (see dashed square in Fig. 6). The dark current parameters of Samples A and B are shown in Table II. We can see a slight reduction in the radiative current for Sample B. The $\beta$ parameters are similar for the two tunnel currents. However, the $\alpha$ parameters of each component are slightly reduced. All these modifications can be attributed to the higher active region growth temperature which is well known to decrease both the residual doping level and the trap density.

The high active region temperature growth of $675^{\circ} \mathrm{C}$ was chosen for the remaining studied epitaxial stacks (C, D, $\mathrm{E}$, and F).

\begin{tabular}{|c|c|c|c|c|c|c|}
\hline Sample & Thickness (nm) & $j_{\text {rad }}^{s}\left(\mathrm{nA} \mathrm{cm}^{-2}\right)$ & $\alpha_{\text {tat }}\left(\mathrm{A} \mathrm{V}^{-2} \mathrm{~m}^{-1}\right)$ & $\beta_{\text {tat }}\left(\mathrm{kV} \mathrm{cm}^{-1}\right)$ & $\alpha_{b 2 b}\left(\mathrm{~A} \mathrm{~V}^{-2} \mathrm{~m}^{-1}\right)$ & $\beta_{b 2 b}\left(\mathrm{kV} \mathrm{cm}^{-1}\right)$ \\
\hline A & 90 & $5.27 \pm 0.02$ & $(2.7 \pm 0.4) \times 10^{-9}$ & $47 \pm 17$ & $2.4 \pm 0.7$ & $(2.6 \pm 0.1) \times 10^{3}$ \\
\hline $\mathrm{B}$ & 90 & $3.67 \pm 0.01$ & $(1.7 \pm 0.4) \times 10^{-9}$ & $47 \pm 22$ & $1.5 \pm 0.3$ & $(2.6 \pm 0.1) \times 10^{3}$ \\
\hline $\mathrm{C}$ & 110 & $2.93 \pm 0.01$ & $(1.7 \pm 0.4) \times 10^{-9}$ & $47 \pm 12$ & $0.11 \pm 0.01$ & $(2.1 \pm 0.1) \times 10^{3}$ \\
\hline $\mathrm{D}$ & 130 & $2.82 \pm 0.01$ & $(7.0 \pm 0.7) \times 10^{-10}$ & $47 \pm 8$ & $0.24 \pm 0.03$ & $(2.1 \pm 0.1) \times 10^{3}$ \\
\hline $\mathrm{E}$ & 150 & $2.21 \pm 0.01$ & $(8.2 \pm 0.7) \times 10^{-10}$ & $42 \pm 6$ & $0.24 \pm 0.03$ & $(1.5 \pm 0.1) \times 10^{3}$ \\
\hline $\mathrm{F}$ & 200 & $1.99 \pm 0.01$ & $(6.1 \pm 0.5) \times 10^{-10}$ & $42 \pm 5$ & $\ldots$ & $\ldots$ \\
\hline
\end{tabular}

TABLE II. Parameters of the three dark current components $j_{\text {rad }}, j_{t a t}$, and $j_{b 2 b}$ for each sample. 


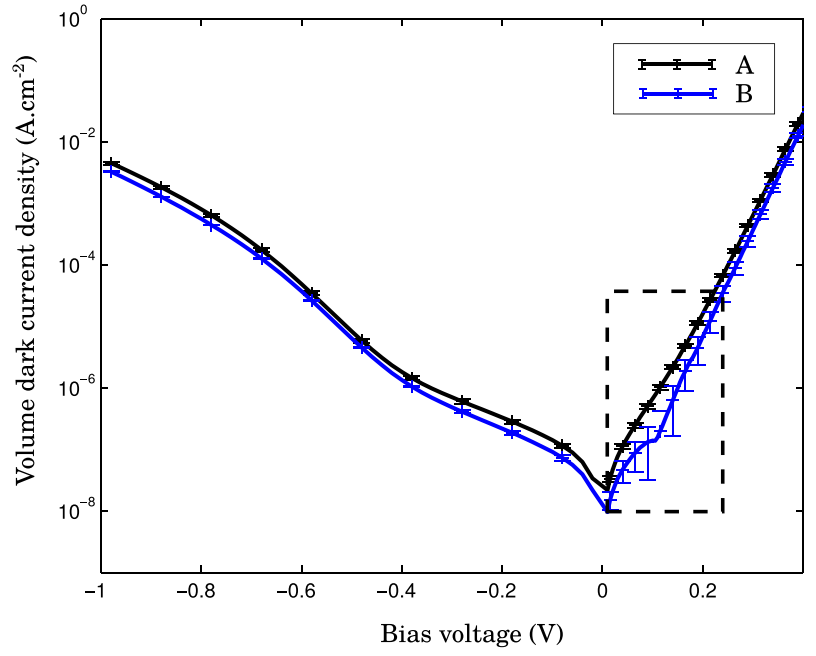

FIG. 6. Volume dark current density of Samples A and B.

\section{Active region thickness}

One of the simplest ways of reducing tunneling effects is to increase the barrier width seen by the carriers. Rather than reducing the doping level in the QNRs, we chose to increase the active region thickness. In this approach, the total semiconductor thickness was kept constant, in order to keep the GMR geometry unchanged (see Samples C, D, E, and $\mathrm{F}$ in Table II). If we maintain $100 \mathrm{~nm}$ of barrier material on each side of the active region, the maximum InGaAs active region thickness is about $200 \mathrm{~nm}$. At resonance, the electric component of the electromagnetic field in the InGaAs absorbing layer was spread over a thickness of less than $100 \mathrm{~nm} .{ }^{16}$ Therefore, the calculated EQE did not significantly change (less than $5 \%$ at $\lambda=1.55 \mu \mathrm{m}$ ) when the active region thickness was increased from $90 \mathrm{~nm}$ to $200 \mathrm{~nm}$ (calculations done without modifying the resonator geometry).

The dark current densities we modeled, as well as the experimental current densities, are plotted in Figure 7. Dark current parameters are reported in Table II.

The variation in $j_{\text {rad }}^{s}$ as a function of active region thickness (see Table II) showed that the dark current arising from

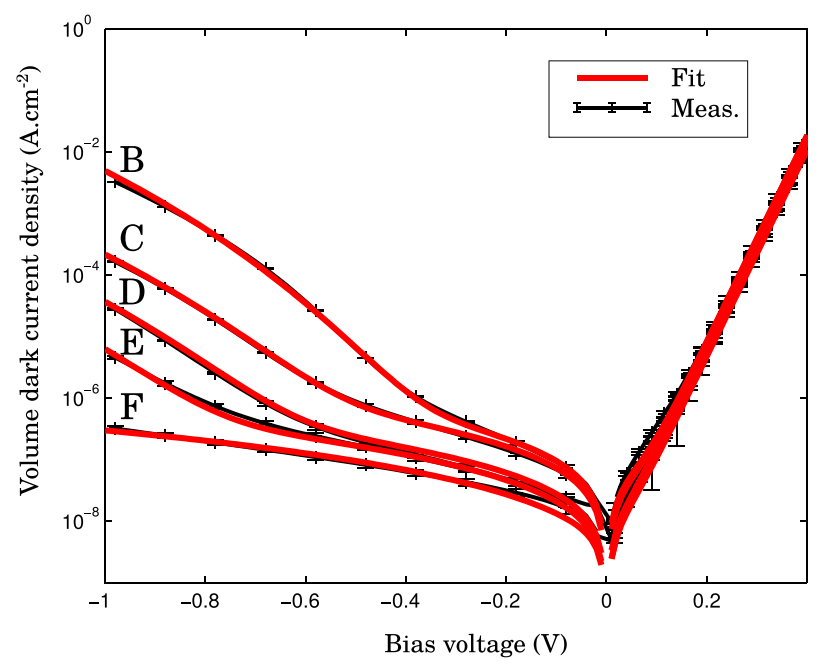

FIG. 7. Volume dark current densities of Samples B, C, D, E, and F. Fitting parameters are shown in Table II.

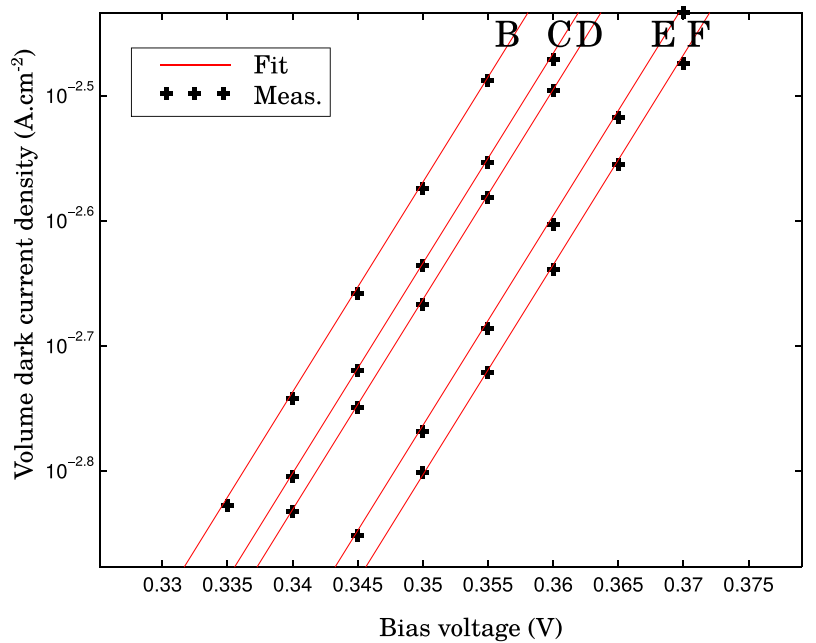

FIG. 8. Volume dark current densities of Samples B, C, D, E, and F for a reduced forward bias voltage range.

the radiative recombination decreases when the thickness of the active region increases. This is well illustrated in Figure 8 for a reduced forward bias voltage range. Our experimental data (see Table II) confirmed Humphreys' radiative lifetime theory ${ }^{33,34}$ mentioned earlier, whereby photon reabsorption becomes more probable as thickness increases, and the radiative lifetime therefore increases linearly. However, to accurate model the radiative lifetime, we had to take the whole stack into account, and in particular, the InGaAs layers. This can be done through numerical simulations, as proposed by Jóźwikowski. ${ }^{35}$

Under reverse bias voltage, the volume dark current density was strongly influenced by active region thickness: it decreased as thickness increased. This confirmed our assumption of tunneling effects. For both band-to-band tunneling and trap-assisted tunneling currents, the $\beta$ parameters were roughly constant whatever the active region thickness. Therefore, the potential barrier height seen by the carriers during the tunneling effects was always the same, whatever the InGaAs layer thickness. $\alpha$ parameters could vary by one order of magnitude. This was due to a mathematical effect: a small variation between $B$ and the other samples was sufficient to induce this huge change in $\alpha$ because of the exponential variation in $\beta$ in the expression of the tunneling current (see Eq. (3)). The only reason why the tunneling currents were reduced as a function of thickness therefore seems to be that the electric field decreased in the active region. By taking an average value for the $\alpha$ and $\beta$ parameters, we could thus retrieve the experimental reverse dark current behavior as a function of the active region thickness (see Fig. 9).

Tunneling effects grow as the thickness of the active layer is reduced. In particular, they strongly predominate in the dark current for InGaAs layers thinner than $100 \mathrm{~nm}$. Therefore, the optimum thickness is the best tradeoff between the $\mathrm{G} / \mathrm{R}$ dark current component and the tunneling current.

\section{OUTLOOK}

In order to reduce the reverse dark current by keeping an active region only $100 \mathrm{~nm}$ thick, the doping profile has to 


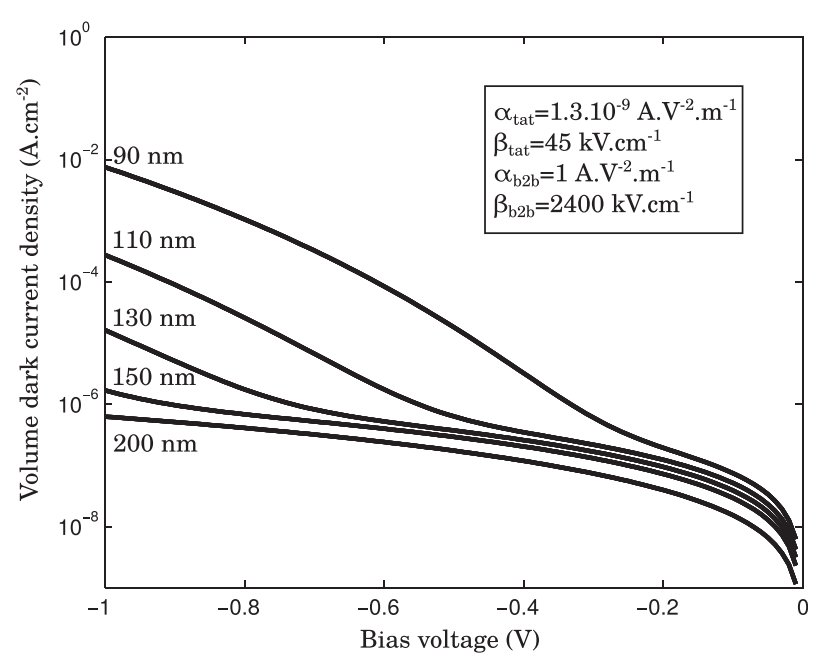

FIG. 9. Calculated reverse volume dark current density for different active region thicknesses. Dark current parameters are shown in inset.

be optimized. By reducing the doping level at the edges of the SCR, the barrier seen by the carriers is widened and fewer states are available for the tunneling current. Furthermore, the trap responsible for the trap-assisted current can be determined by deep-level transient spectroscopy (DLTS). Once the trap energy levels have been determined, their densities can be reduced.

Today, the major contribution to dark current comes from peripheral dark current leakage due to mesa etching. Technological efforts are currently underway to reduce this component. Assuming that we are able to drastically reduce it, Sample F would yield a total dark current density of $20 \mathrm{nA} \mathrm{cm}^{-2}$ at room temperature for a reverse bias $U=100 \mathrm{mV}$.

As the EQE does not depend much on the active region thickness, owing to the electric field enhancement, we can use the external quantum efficiency shown in Figure 2 to calculate the specific detectivity,

$$
D^{*}=\sqrt{q} \frac{\lambda}{h c} \frac{\eta(\lambda)}{\sqrt{2 j_{\text {dark }}}},
$$

where $\eta$ is the external quantum efficiency. The numerical calculation yields $D^{*}=1 \times 10^{13} \mathrm{~cm} \sqrt{\mathrm{Hz}} \mathrm{W}^{-1}$ for $\lambda=1.55$ $\mu \mathrm{m}$, a value close to that of today's best devices. ${ }^{7-9}$ This is the maximum detectivity we can typically achieve with our structure. To go further and achieve still better performances, growth conditions will have to be improved, in order to reduce traps and determine another tradeoff with a thinner active region.

\section{CONCLUSION}

The state of the art for InGaAs photodiode structures obtained by $\mathrm{Zn}$ diffusion has certainly surpassed the expectations that its designers had more than 30 years ago. Any further improvements, however, can only be marginal, and will have to be achieved through lengthy optimizations of the geometry of the FPA.
Optical nano-resonators allow junction thickness to be substantially diminished, all the while maintaining high absorption across a broad spectral band. In this context, we can introduce heterojunctions at each edge of the active region, to drastically reduce diffusion currents. The SCR thickness can be reduced by more than an order of magnitude, reducing the generation/recombination current by the same extent.

In this new context, epitaxial growth must be completely rethought. We implemented a characterization method that allowed us to study volume current with considerable precision.

Our experimental study of MOVPE thin junctions showed that tunneling currents in reverse bias voltages increase when active region thickness decreases. There is, therefore, an optimum thickness (a few hundred nanometers for our structure) that represents the best compromise between reducing the generation/recombination current and increasing tunnel currents.

In forward bias voltages, radiative lifetime is reduced as the thickness decreases, because of the so-called photon recycling effect. This effect could be particularly important for photovoltaic applications where we try to reduce radiative recombination.

Our investigation of epitaxial stack optimization in six structures shows that we are already able to achieve state-ofthe-art detectivity.

This study paves the way for a fourth generation of InGaAs photodiodes featuring a nano-resonator and a double heterojunction photodiode.

\section{ACKNOWLEDGMENTS}

This work was supported by a DGA-MRIS scholarship, the French RENATECH network, and the NightGlow research program funded by ONERA.

\footnotetext{
${ }^{1}$ A. Rogalski, Infrared Detectors (CRC Press, 2010).

${ }^{2}$ M. A. Kinch, State-of-the-Art Infrared Detector Technology (SPIE Press, 2014).

${ }^{3}$ P. Martyniuk, J. Antoszewski, M. Martyniuk, L. Faraone, and A. Rogalski, "New concepts in infrared photodetector designs," Appl. Phys. Rev. 1, 041102 (2014).

${ }^{4}$ M. P. Hansen and D. S. Malchow, "Overview of swir detectors, cameras, and applications," Proc. SPIE 6939, 69390I (2008).

${ }^{5}$ S. Derelle, P. Simoneau, J. Deschamps, S. Rommeluère, M. Hersé, G. Moreels, E. De Borniol, and O. Pacaud, "Development of low-flux swir radio-imaging systems to study nightglow emission," Proc. SPIE 8353, 83533P (2012).

${ }^{6} \mathrm{~A}$. Rogalski and R. Ciupa, "Performance limitation of short wavelength infrared InGaAs and $\mathrm{HgCdTe}$ photodiodes," J. Electron. Mater. 28, 630-636 (1999).

${ }^{7}$ M. MacDougal, J. Geske, C. Wang, S. Liao, J. Getty, and A. Holmes, "Low dark current InGaAs detector arrays for night vision and astronomy," Proc. SPIE 7298, 72983F (2009).

${ }^{8}$ J. A. Trezza, N. Masaun, and M. Ettenberg, "Analytic modeling and explanation of ultra-low noise in dense swir detector arrays," in Proc. SPIE 8012, 80121Y (2011).

${ }^{9}$ A. Rouvié, J.-L. Reverchon, O. Huet, A. Djedidi, J.-A. Robo, J.-P. Truffer, T. Bria, M. Pires, J. Decobert, and E. Costard, "InGaAs focal plane array developments at iii-v lab," in Proc. SPIE 8353, 835308 (2012).

${ }^{10}$ B. Sverdlov, A. Botchkarev, N. Teraguchi, A. Salvador, and H. Morkoç, "Reduction of dark current in photodiodes by the use of a resonant cavity," Electron. Lett. 29, 1019-1021 (1993).
} 
${ }^{11}$ A. Dentai, R. Kuchibhotla, J. C. Campbell, C.-Y. Tsai, and C. Lei, "High quantum efficiency, long wavelength $\mathrm{InP} / \mathrm{InGaAs}$ microcavity photodiode," Electron. Lett. 27, 2125-2127 (1991).

${ }^{12}$ I.-H. Tan, E. L. Hu, J. Bowers, and B. Miller, "Modeling and performance of wafer-fused resonant-cavity enhanced photodetectors," IEEE J. Quantum Electron. 31, 1863-1875 (1995).

${ }^{13}$ A. Salvador, B. Sverdlov, T. Lehner, A. Botchkarev, F. Huang, and H. Morkoc, "Resonant cavity enhanced InP/InGaAs photodiode on Si using epitaxial liftoff," Appl. Phys. Lett. 65, 1880-1882 (1994).

${ }^{14}$ M. S. Ünlü and S. Strite, "Resonant cavity enhanced photonic devices," J. Appl. Phys. 78, 607-639 (1995).

${ }^{15}$ P. Bouchon, F. Pardo, R. Haïdar, and J.-L. Pelouard, "Fast modal method for subwavelength gratings based on b-spline formulation," JOSA A $\mathbf{2 7}$, 696-702 (2010).

${ }^{16}$ M. Verdun, B. Portier, K. Jaworowicz, J. Jaeck, F. Lelarge, S. Guilet, C. Dupuis, R. Haïdar, F. Pardo, and J.-L. Pelouard, "Guided-mode resonator for thin InGaAs pin short-wave infrared photo-diode," Appl. Phys. Lett. 108, 053501 (2016).

${ }^{17}$ O. K. Kim, B. V. Dutt, R. McCoy, and J. R. Zuber, "A low dark-current, planar InGaAs pin photodiode with a quaternary InGaAsP cap layer," IEEE J. Quantum Electron. 21, 138-143 (1985).

${ }^{18}$ S. Miura, H. Kuwatsuka, T. Mikawa, and O. Wada, "Planar, embedded InP/GaInAs p-i-n photodiode with very high-speed response characteristics," Appl. Phys. Lett. 49, 1522-1524 (1986).

${ }^{19}$ M. Gallant, N. Puetz, A. Zemel, and F. Shepherd, "Metalorganic chemical vapor deposition InGaAs p-i-n photodiodes with extremely low dark current,” Appl. Phys. Lett. 52, 733-735 (1988).

${ }^{20}$ S. Kagawa, K. Inoue, I. Ogawa, Y. Takada, and T. Shibata, "Wide-wavelength InGaAs/inp pin photodiodes sensitive from 0.7 to $1.6 \mu \mathrm{m}$," Jpn. J. Appl. Phys. 28, 1843 (1989).

${ }^{21}$ A. R. Wichman, R. E. DeWames, and E. Bellotti, "Three-dimensional numerical simulation of planar $\mathrm{p}+\mathrm{n}$ heterojunction $\mathrm{In}_{0.53} \mathrm{Ga}_{0.47}$ as photodiodes in dense arrays part I: Dark current dependence on device geometry," Proc. SPIE 9070, 907003 (2014).

${ }^{22}$ I. Vurgaftman, J. Meyer, and L. Ram-Mohan, "Band parameters for iii-v compound semiconductors and their alloys," J. Appl. Phys. 89, 5815-5875 (2001).
${ }^{23}$ J. Decobert and G. Patriarche, "Transmission electron microscopy study of the inp/InGaAs and InGaAs/inp heterointerfaces grown by metalorganic vapor-phase epitaxy," J. Appl. Phys. 92, 5749-5755 (2002).

${ }^{24}$ C. Blaauw, F. Shepherd, and D. Eger, "Secondary ion mass spectrometry and electrical characterization of $\mathrm{Zn}$ diffusion in n-type InP," J. Appl. Phys. 66, 605-610 (1989).

${ }^{25}$ F. Reier, N. Agrawal, P. Harde, and R. Bochnia, "Highly abrupt modulation $\mathrm{Zn}$ doping in LP-MOVPE grown InAlAs as applied to quantum well electron transfer structures for optical switching," in Proceedings of the Fifth International Conference on Indium Phosphide and Related Materials (IEEE, 1993), pp. 703-706.

${ }^{26}$ H. Kim, J. Choi, H. Bang, Y. Jee, S. Yun, J. Burm, M. Kim, and A. Choo, "Dark current reduction in APD with BCB passivation," Electron. Lett. 37(7), 455 (2001).

${ }^{27} \mathrm{R}$. Trommer and H. Albrecht, "Confirmation of tunneling current via traps by dlts measurements in InGaAs photodiodes," Jpn. J. Appl. Phys. 22, L364 (1983).

${ }^{28}$ E. O. Kane, "Theory of tunneling," J. Appl. Phys. 32, 83-91 (1961).

${ }^{29}$ W. Loke, S. Yoon, S. Wicaksono, K. Tan, and K. Lew, "Defect-induced trap-assisted tunneling current in GaInNAs grown on GaAs substrate," J. Appl. Phys. 102, 054501 (2007).

${ }^{30}$ G. Hurkx, D. Klaassen, and M. Knuvers, "A new recombination model for device simulation including tunneling," IEEE Trans. Electron Devices 39, 331-338 (1992)

${ }^{31}$ W. Van Roosbroeck and W. Shockley, "Photon-radiative recombination of electrons and holes in germanium," Phys. Rev. 94, 1558 (1954).

${ }^{32}$ E. Zielinski, H. Schweizer, K. Streubel, H. Eisele, and G. Weimann, "Excitonic transitions and exciton damping processes in InGaAs/InP," J. Appl. Phys. 59, 2196-2204 (1986).

${ }^{33} \mathrm{R}$. Humphreys, "Radiative lifetime in semiconductors for infrared detection," Infrared Phys. 23, 171-175 (1983).

${ }^{34}$ R. Humphreys, "Radiative lifetime in semiconductors for infrared detection," Infrared Phys. 26, 337-342 (1986).

${ }^{35}$ K. Jóźwikowski, M. Kopytko, and A. Rogalski, "Numerical estimations of carrier generation-recombination processes and the photon recycling effect in $\mathrm{HgCdTe}$ heterostructure photodiodes," J. Electron. Mater. 41, 2766-2774 (2012). 